\title{
Reactor de membrana multitubular para la producción de hidrógeno: guía de diseño
}

\author{
Multi-tubular membrane reactor for hydrogen production: design guide.
}

\author{
Luis Eduardo Maya Vinueza \\ Ingeniero químico, Universidad Nacional de Colombia, Sede Manizales, Manizales, Colombia \\ Correo-e: lemayav@unal.edu.co
}

\begin{abstract}
Resumen- El presente trabajo analiza el rendimiento de un reactor de membrana multitubular para la producción de hidrógeno con la reacción water-gas shift. Las condiciones de operación se definen considerando que el sistema se encuentra acoplado con un reformado de etanol y celdas de combustible para la producción de energía. Usando un modelo que considera solo dirección axial y estado estable, se presentan varias herramientas de diseño con el fin de lograr conversiones altas mediante la variación de la presión en el lado de la reacción y el número de tubos. Finalmente, el análisis de diferentes configuraciones de reactores, ayudó a concluir que la conversión de monóxido de carbono cambia proporcionalmente con aumentos de presión en la coraza y el número de tubos, que se explica por el incremento en la permeación de hidrógeno.
\end{abstract}

Palabras clave-Guía de diseño, producción de hidrogeno, reactor de membrana multitubular, Water-gas shift.

Abstract- The present work analyzes the multi-tubular membrane reactor yield in order to produce hydrogen through water-gas shift reaction. The operative conditions were defined considering the process joined with reforming of ethanol and fuel cells for energy production. Using a model which considers only axial direction and steady state is presented multiple design tools, with the purpose of reaching high values of conversion by changing the pressure in the reaction side and number of tubes. Finally, the analysis of different reactor configurations helped to deduce that carbon monoxide conversion, changes proportionally with increases in the pressure in the shell and number of tubes, which can be explained due to increments in hydrogen permeation.

Key Word - Design guide, hydrogen production, multi-tubular membrane reactor, Water-gas shift.

\section{INTRODUCCIÓN}

En los últimos años, la intensificación de procesos ha alcanzado un papel importante en la Ingeniería Química, debido a su potencial como herramienta para el mejoramiento de procesos de producción existentes, proporcionando instrumentos para la formulación de guías de diseño que involucran el acoplamiento de operaciones [1]. Uno de estos casos es el reactor de membrana (RM), el cual posee un gran potencial, puesto que incrementa la conversión de la reacción de interés, lo que significa una reducción significativa en el tamaño de planta así como un mejor aprovechamiento de materias primas al compararse con un reactor convencional (RC).

Uno de los casos de mayor estudio que involucra la tecnología de membrana es la producción de hidrógeno. Actualmente, según Francesconi et al. [2] se ha proporcionado bastante interés sobre la reacción water-gas shift (WGS), puesto que puede ser acoplada con el reformado de alcoholes (e.g. etanol) y celdas de combustible para la producción de calor y energía (e.g. PEMFC). En la ecuación (1) se presenta la reacción WGS.

$\mathrm{CO}+\mathrm{H}_{2} \mathrm{O} \leftrightarrow \mathrm{CO}_{2}+\mathrm{H}_{2} \quad \Delta \mathrm{H}_{\mathrm{rxn}}^{298 \mathrm{~K}}=-41.09 \mathrm{~kJ} / \mathrm{mol}$

La reacción se caracteriza por su exotermicidad y por no poseer cambio en el número de moles, lo que indica que la presión no tiene efecto para el caso de un reactor convencional.

El modelo utilizado para el RM posee una configuración multitubular dentro de una coraza. Se utiliza un catalizador de óxido de hierro y óxido de cromo, el cual es empacado en el lado de la coraza, mientras que el hidrógeno permea desde este hacia los tubos. La membrana de paladio soportada sobre sustratos cerámicos ofrece alta selectividad para la permeación de hidrógeno [3], de manera que provee disminuciones en el costo del reactor (se requiere menor volumen de catalizador con respecto a un RC). Para este caso, se prefiere un aumento considerable en la presión del retentato manteniendo una presión de trabajo en el permeato de 1 atmósfera, lo que permite un aumento del gradiente de concentración de hidrógeno y por lo tanto de la permeación del mismo.

A través del análisis de las fronteras y de las condiciones de operación, se proponen herramientas conceptuales con el fin de manipular el sistema, y así, incrementar la conversión del monóxido de carbono y la producción de hidrógeno. Se utiliza para ello, la metodología de equilibrio termodinámico de RM de Rezai y Traa [4], y las guías de diseño propuestas por Moon y Park [5]. 


\section{CONTENIDO}

\section{A. Modelo matemático.}

En la Fig. 1 se muestra el esquema del reactor multitubular.

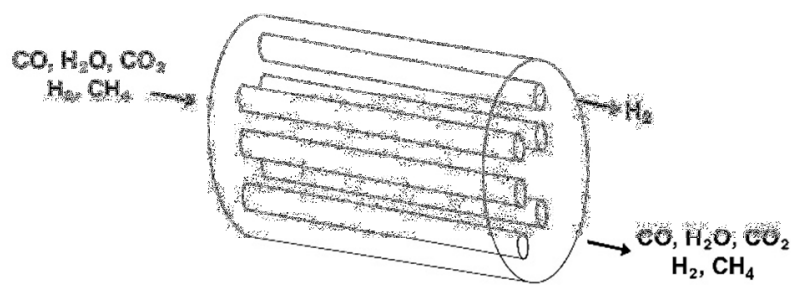

Figura 1. Esquema del reactor de membrana.

Basándose en el supuesto de que se requiere la producción de $10 \mathrm{~kW}$ en una celda de combustible, el flujo de salida en el reformado de etanol sería de $345 \mathrm{~mol} / \mathrm{h}$, donde las composiciones vienen dadas por la Tabla 1, según Adrover et al. [6].

\begin{tabular}{|c|c|}
\hline Componente & Fracción Molar \\
\hline Monóxido de Carbono & 0.0797 \\
\hline Agua & 0.3188 \\
\hline Dióxido de Carbono & 0.1099 \\
\hline Hidrógeno & 0.4348 \\
\hline Metano & 0.0568 \\
\hline
\end{tabular}

Tabla 1. Composiciones de la corriente de alimento al RM.

El análisis del sistema planteado se aborda inicialmente con la determinación de sus respectivas fronteras, lo cual se lleva a cabo analizando el sistema en su equilibrio termodinámico [4]. Para ello, se plantean los balances molares tanto para el retentato como para el permeato considerando tanto el equilibrio químico (reacción) y el equilibrio del componente que permea (hidrógeno). Los balances se muestran en la Tabla 2.

El sistema se resuelve considerando que la actividad del componente que permea es igual en el lado de la reacción así como del lado del permeato (ver ecuación 2):

$$
\left(\mathrm{a}_{\mathrm{H}_{2}}\right)^{\mathrm{R}}=\left(\mathrm{a}_{\mathrm{H}_{2}}\right)^{\mathrm{P}}
$$

La constante de equilibrio se calcula como se indica en la ecuación (3):

$$
\ln \left(\mathrm{K}_{\mathrm{eq}}\right)=-\frac{\Delta \mathrm{G}_{\mathrm{rxn}}}{\mathrm{RT}^{\mathrm{R}}}
$$

La constante de equilibrio por definición, es igual a la productoria de las actividades elevadas a su coeficiente estequiométrico (ver ecuación 4).

$$
\mathrm{K}_{\mathrm{eq}}=\left(\prod_{\mathrm{j}=1}^{\mathrm{N}} \mathrm{a}_{\mathrm{j}}^{\mathrm{v}_{\mathrm{j}}}\right)^{\mathrm{R}}
$$

El reactor de membrana se modela teniendo en cuenta tan solo la dirección axial y en estado estable bajo una serie de supuestos:

- La caída de presión es despreciable (condiciones isobáricas).

- La membrana es altamente selectiva al hidrógeno.

- Flujo en co-corriente.

- El reactor es adiabático con respecto a los alrededores (ambiente).

Los balances molares y de energía se presentan a continuación.

- $\quad$ Retentato (Coraza).

Balances molares por componente.

$$
\begin{aligned}
& \frac{\mathrm{dF}_{\mathrm{CO}}^{\mathrm{R}}}{\mathrm{dz}}=\mathrm{A}_{\mathrm{T}}\left(\mathrm{r}_{\mathrm{CO}}\right) \rho_{\mathrm{B}} \\
& \frac{\mathrm{dF}_{\mathrm{H}_{2} \mathrm{O}}^{\mathrm{O}}}{\mathrm{dz}}=\mathrm{A}_{\mathrm{T}}\left(\mathrm{r}_{\mathrm{CO}}\right) \rho_{\mathrm{B}} \\
& \frac{\mathrm{dF}_{\mathrm{CO}_{2}}^{\mathrm{R}}}{\mathrm{dz}}=\mathrm{A}_{\mathrm{T}}\left(\mathrm{r}_{\mathrm{CO}}\right) \rho_{\mathrm{B}} \\
& \frac{\mathrm{dF}_{\mathrm{H}_{2}}^{\mathrm{R}}}{\mathrm{dz}}=\mathrm{A}_{\mathrm{T}}\left(-\mathrm{r}_{\mathrm{CO}}\right) \rho_{\mathrm{B}}-\pi \mathrm{d}_{\mathrm{te}} \mathrm{J}_{\mathrm{H}_{2}} \mathrm{~N}_{\mathrm{t}}
\end{aligned}
$$

Balance de energía

$$
\frac{\mathrm{dT}^{\mathrm{R}}}{\mathrm{dz}}=\frac{\mathrm{A}_{\mathrm{T}} \rho_{\mathrm{B}}\left(-\mathrm{r}_{\mathrm{CO}}\right)\left(-\Delta \mathrm{H}_{\mathrm{rxn}}\right)-\pi \mathrm{N}_{\mathrm{T}} \mathrm{d}_{\mathrm{ti}} \mathrm{U}\left(\mathrm{T}^{\mathrm{R}}-\mathrm{T}^{\mathrm{P}}\right)-\pi \mathrm{d}_{\mathrm{te}} \mathrm{J}_{\mathrm{H}_{2}} \mathrm{~N}_{\mathrm{t}} \mathrm{C}_{\mathrm{pH}}^{\mathrm{R}}}{\sum_{\mathrm{j}=1}^{\mathrm{N}} \mathrm{F}_{\mathrm{j}}^{\mathrm{R}} \mathrm{C}_{\mathrm{pj}}^{\mathrm{R}}}
$$

- Permeato (Tubos).

Balances molares por componente.

$$
\frac{\mathrm{dF}_{\mathrm{H}_{2}}^{\mathrm{P}}}{\mathrm{dz}}=\pi \mathrm{d}_{\mathrm{te}} \mathrm{J}_{\mathrm{H}_{2}} \mathrm{~N}_{\mathrm{t}}
$$

Balance de energía.

$$
\frac{\mathrm{dT}^{\mathrm{P}}}{\mathrm{dz}}=\frac{\pi \mathrm{d}_{\mathrm{te}} \mathrm{J}_{\mathrm{H}_{2}} \mathrm{~N}_{\mathrm{t}} \mathrm{C}_{\mathrm{pH}_{2}}^{\mathrm{R}}+\pi \mathrm{N}_{\mathrm{T}} \mathrm{d}_{\mathrm{ti}} \mathrm{U}\left(\mathrm{T}^{\mathrm{R}}-\mathrm{T}^{\mathrm{P}}\right)}{\mathrm{F}_{\mathrm{H}_{2}}^{\mathrm{P}} \mathrm{C}_{\mathrm{pH}}^{\mathrm{P}}}
$$




\begin{tabular}{|c|c|c|c|c|c|}
\hline Lado & Especie & Inicial & Cambio & Remanentes & Fracción Molar \\
\hline \multirow{5}{*}{ Reacción } & Monóxido de Carbono & $N_{C O, 0}$ & $-N_{C O, 0} X_{1}$ & $N_{C O, 0}\left(1-X_{1}\right)$ & $\frac{N_{C O, 0}\left(1-X_{1}\right)}{N_{T, 0}-N_{C O, 0} X_{2}}$ \\
\cline { 2 - 6 } & Agua & $N_{H_{2} O, 0}$ & $-N_{C O, 0} X_{1}$ & $N_{H_{2} O, 0}-N_{C O, 0} X_{1}$ & $\frac{N_{H_{2} O, 0}-N_{C O, 0} X_{1}}{N_{T, 0}-N_{C O, 0} X_{2}}$ \\
\cline { 2 - 6 } & Dióxido de Carbono & $N_{C O_{2}, 0}$ & $N_{C O, 0} X_{1}$ & $N_{C O_{2}, 0}+N_{C O, 0} X_{1}$ & $\frac{N_{C O_{2}, 0}+N_{C O, 0} X_{1}}{N_{T, 0}-N_{C O, 0} X_{2}}$ \\
\cline { 2 - 6 } & Hidrógeno & $N_{H_{2}, 0}$ & $N_{C O, 0} X_{1}-N_{C O, 0} X_{2}$ & $N_{H_{2}, 0}+N_{C O, 0}\left(X_{1}-X_{2}\right)$ & $\frac{N_{H_{2}, 0}+N_{C O, 0}\left(X_{1}-X_{2}\right)}{N_{T, 0}-N_{C O, 0} X_{2}}$ \\
\cline { 2 - 6 } & Metano & $N_{C H_{4}, 0}$ & 0 & $N_{C H_{4}, 0}$ & $\frac{N_{C, 0}}{N_{T, 0}-N_{C O, 0} X_{2}}$ \\
\cline { 2 - 6 } Permeato & Total & $N_{T, 0}$ & $-N_{C O, 0} X_{2}$ & $N_{T, 0}-N_{C O, 0} X_{2}$ & 1 \\
\hline & Hidrógeno & 0 & $N_{C O, 0} X_{2}$ & $N_{C O, 0} X_{2}$ & 1 \\
\cline { 2 - 6 } & Total & 0 & $N_{C O, 0} X_{2}$ & $N_{C O, 0} X_{2}$ & 1 \\
\hline
\end{tabular}

Tabla 2. Balances molares para la reacción WGS.

Se utiliza la expresión de velocidad de reacción propuesta por Podolski y Kim [7], basada en el modelo de LangmuirHinshelwood, para un catalizador de óxido de hierro (93\%) y óxido de cromo (7\%), con un diámetro de partícula de $1 \mathrm{~mm}$.

$$
-\mathrm{r}_{\mathrm{CO}}=\frac{\mathrm{kK}_{\mathrm{CO}} \mathrm{K}_{\mathrm{H}_{2} \mathrm{O}}\left(\mathrm{P}_{\mathrm{CO}}^{\mathrm{R}} \mathrm{P}_{\mathrm{H}_{2} \mathrm{O}}^{\mathrm{R}}-\mathrm{P}_{\mathrm{CO}_{2}}^{\mathrm{R}} \mathrm{P}_{\mathrm{H}_{2}}^{\mathrm{R}} \frac{1}{\mathrm{~K}_{\mathrm{eq}}}\right)}{\left(1+\mathrm{K}_{\mathrm{CO}} \mathrm{P}_{\mathrm{CO}}^{\mathrm{R}}+\mathrm{K}_{\mathrm{H}_{2}} \mathrm{O}_{\mathrm{H}_{2} \mathrm{O}}^{\mathrm{R}}+\mathrm{K}_{\mathrm{CO}_{2}} \mathrm{P}_{\mathrm{CO}_{2}}^{\mathrm{R}}\right)^{2}}
$$

La permeación a través de la membrana de Paladio soportada sobre $\gamma$-Alúmina se expresa en términos de la ley de Sievert propuesta por Scura et al. [8].

$$
\mathrm{J}_{\mathrm{H}_{2}}=\frac{\mathrm{P}_{\mathrm{e} 0} \mathrm{e}^{-\mathrm{E} / \mathrm{RT}^{\mathrm{R}}}}{\delta}\left(\sqrt{\mathrm{P}_{\mathrm{H}_{2}}^{\mathrm{R}}}-\sqrt{\mathrm{P}_{\mathrm{H}_{2}}^{\mathrm{P}}}\right)
$$

En el presente trabajo se usa las características de una membrana comercial con espesor de $6 \mu \mathrm{m}$, y los siguientes parámetros de la misma [9].

$$
\begin{gathered}
\mathrm{P}_{\mathrm{e} 0}=970 \frac{\mathrm{nmol}}{\mathrm{ms} \mathrm{Pa}^{0.5}} \\
\frac{\mathrm{E}}{\mathrm{R}}=3098 \mathrm{~K}
\end{gathered}
$$

El coeficiente global de transferencia de calor se calcula según Kern [10].

$$
\mathrm{U}=\frac{1}{\frac{1}{\mathrm{~h}^{\mathrm{P}}+\frac{1 \mathrm{~d}_{\mathrm{ti}}}{\mathrm{h}^{\mathrm{R}} \mathrm{d}_{\mathrm{te}}}}}
$$

Donde los coeficientes convectivos de transferencia de calor $h^{\mathrm{P}} \mathrm{y} \mathrm{h}^{\mathrm{R}}$, fueron calculados según Kern, y Froment y Bischoff [11], respectivamente.
Se utiliza la metodología propuesta por Moon y Park para identificar los efectos de la permeación y la velocidad de reacción con respecto a la conversión de salida del reactor de membrana, de manera que se provea una guía de diseño. A continuación se presentan los balances para coraza y tubos expresados en función de los parámetros adimensionales de Damköehler (Da) y Peclet (Pe).

- Retentato (Coraza).

Balances adimensionales por componente.

$$
\begin{aligned}
& \frac{\mathrm{dY}_{\mathrm{CO}}^{\mathrm{R}}}{\mathrm{d} \xi}=-\mathrm{nD}_{\mathrm{a}} \\
& \frac{\mathrm{dY}_{\mathrm{H}_{2} \mathrm{O}}^{\mathrm{R}}}{\mathrm{d} \xi}=-\mathrm{nD}_{\mathrm{a}} \\
& \frac{\mathrm{dY}_{\mathrm{CO}_{2}}^{\mathrm{R}}}{\mathrm{d} \xi}=\mathrm{nD}_{\mathrm{a}}
\end{aligned}
$$$$
\frac{d Y_{\mathrm{H}_{2}}^{\mathrm{R}}}{\mathrm{d} \xi}=\mathrm{nD}_{\mathrm{a}}-\frac{1}{\mathrm{Pe}} \mathrm{N}_{\mathrm{t}}\left(\sqrt{\frac{\mathrm{Y}_{\mathrm{H}}^{\mathrm{R}}}{\sum \mathrm{Y}_{\mathrm{i}}^{\mathrm{R}}}}-\sqrt{\frac{\mathrm{Y}_{\mathrm{H}_{2}}^{\mathrm{P}}}{\sum \mathrm{Y}_{\mathrm{i}}^{\mathrm{P}}}} \sqrt{\frac{\mathrm{P}^{\mathrm{P}}}{\mathrm{P}^{\mathrm{R}}}}\right)
$$

Balance adimensional de energía.

$$
\begin{aligned}
& \frac{d T^{R}}{d \xi}=D a\left(\frac{\left(-\Delta H_{r x n}\right) n}{\sum_{j=1}^{N} Y_{j}^{R} C_{p j}^{R}}\right)-D a\left(\frac{\pi d_{t i} U\left(T^{R}-T^{P}\right) N_{t}}{\rho_{B} k_{H_{2}, 0} P^{R} A_{T} \sum_{j=1}^{N} Y_{j}^{R} C_{p j}^{R}}\right)- \\
& \frac{1}{P e}\left(\frac{C_{p, H_{2}}^{R} N_{t}}{\sum_{j=1}^{N} Y_{j}^{R} C_{p j}^{R}}\left(\sqrt{\frac{Y_{H_{2}}^{R}}{\sum Y_{i}^{R}}}-\sqrt{\frac{Y_{H_{2}}^{P}}{\sum Y_{i}^{P}}} \sqrt{\frac{P^{P}}{P^{R}}}\right)\right)
\end{aligned}
$$

- Permeato (Tubos).

Balances adimensionales por componente. 


$$
\frac{d Y_{\mathrm{H}_{2}}^{\mathrm{P}}}{\mathrm{d} \xi}=\frac{1}{\mathrm{Pe}} \mathrm{N}_{\mathrm{t}}\left(\sqrt{\frac{\mathrm{Y}_{\mathrm{H}_{2}}^{\mathrm{R}}}{\sum \mathrm{Y}_{\mathrm{i}}^{\mathrm{R}}}}-\sqrt{\frac{\mathrm{Y}_{\mathrm{H}_{2}}^{\mathrm{P}}}{\sum \mathrm{Y}_{\mathrm{i}}^{\mathrm{P}}}} \sqrt{\frac{\mathrm{P}^{\mathrm{P}}}{\mathrm{P}^{\mathrm{R}}}}\right)
$$

Balance adimensional de energía.

$$
\begin{aligned}
& \frac{\mathrm{dT}^{\mathrm{P}}}{\mathrm{d} \xi}= \\
& \frac{1}{\mathrm{Pe}}\left(\frac { \mathrm { C } _ { \mathrm { p } , \mathrm { H } _ { 2 } } ^ { \mathrm { R } } } { \mathrm { C } _ { \mathrm { p } , \mathrm { H } _ { 2 } } ^ { \mathrm { P } } } \frac { \mathrm { N } _ { \mathrm { t } } } { \mathrm { Y } _ { \mathrm { H } _ { 2 } } ^ { \mathrm { P } } } \left(\sqrt{\frac{\mathrm{Y}_{\mathrm{H}_{2}}^{\mathrm{R}}}{\sum \mathrm{Y}_{\mathrm{i}}^{\mathrm{R}}}}-\sqrt{\left.\left.\frac{\mathrm{Y}_{\mathrm{H}_{2}}^{\mathrm{P}}}{\sum \mathrm{Y}_{\mathrm{i}}^{\mathrm{P}}} \sqrt{\frac{\mathrm{P}^{\mathrm{P}}}{\mathrm{P}^{\mathrm{R}}}}\right)\right)+}\right.\right. \\
& \operatorname{Da}\left(\frac{\pi \mathrm{d}_{\mathrm{ti}} \mathrm{U}\left(\mathrm{T}^{\mathrm{R}}-\mathrm{T}^{\mathrm{P}}\right) \mathrm{N}_{\mathrm{t}}}{\rho_{\mathrm{B}} \mathrm{k}_{\mathrm{H}_{2}, 0} \mathrm{P}^{\mathrm{R}} \mathrm{A}_{\mathrm{T}} \mathrm{Y}_{\mathrm{H}_{2}}^{\mathrm{P}} \mathrm{C}_{\mathrm{p}, \mathrm{H}_{2}}^{\mathrm{P}}}\right)
\end{aligned}
$$

Se definen los números de Damköehler (Da) y Peclet $(\mathrm{Pe})$ en las ecuaciones (24) y (25), respectivamente.

$$
\begin{gathered}
\mathrm{Da}=\frac{\mathrm{A}_{\mathrm{T}} \mathrm{k}_{\mathrm{H}_{2}, 0} \mathrm{P}^{\mathrm{R}} \rho_{\mathrm{B}} \mathrm{L}}{\mathrm{F}_{\mathrm{H}_{2}, 0}^{\mathrm{R}}} \\
\mathrm{Pe}=\frac{\mathrm{F}_{\mathrm{H}_{2,0}}^{\mathrm{R}}}{\pi \mathrm{Ld}_{\mathrm{te}}\left(\mathrm{P}_{\mathrm{e} 0} / \delta\right) \mathrm{e}^{-\mathrm{E} / \mathrm{RT}^{\mathrm{R}} \sqrt{\mathrm{P}^{\mathrm{R}}}}}
\end{gathered}
$$

Donde Damköehler muestra la relación entre la velocidad de reacción y el transporte convectivo en el retentato, mientras que Peclet indica la relación con respecto a la velocidad de permeación.

\section{B. Resultados y discusión.}

El análisis del reactor de membrana se aborda analizando las fronteras del sistema, lo cual se logra resolviendo las ecuaciones planteadas en la Tabla 2 junto a las correlaciones (2), (3) y (4). El cálculo de las actividades de cada componente se obtiene con la ecuación de estado de PengRobinson. Los resultados se muestran en la Fig. 2.

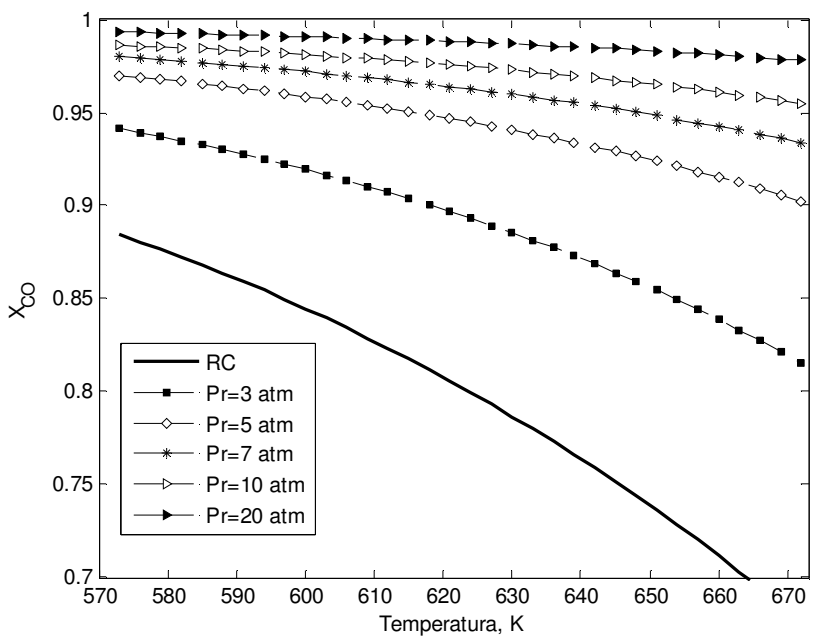

Figura 2. Conversión de equilibrio del monóxido de carbono contra temperatura a diferentes presiones de retentato para un RM y un RC.
La disminución de la conversión de $\mathrm{CO}$ con aumentos de temperatura se debe a la naturaleza de la reacción (exotérmica). Se observan valores de conversión bastante altos para el reactor de membrana (mayores al 93\%), que aumentan a medida que se incrementa la presión en el lado de reacción. Los aumentos en la presión en el retentato permiten que el gradiente de concentración para el hidrógeno aumente significativamente y por lo tanto el equilibrio se desplace hacia los productos, debido a la permeación del mismo. Con respecto al reactor convencional ( $\mathrm{RC}$ ) la conversión no supera el $90 \%$. Dado que la reacción no posee cambio en el número de moles entre reactivos y productos, el efecto de la presión es insignificante, por lo que se analiza a un valor de presión cualquiera.

Para tener un criterio de evaluación para el desempeño del reactor de membrana, se analizarán las siguientes variables:

- Conversión del monóxido de carbono, el cual se define mediante la ecuación (26).

$$
\mathrm{X}_{\mathrm{CO}}=\frac{\mathrm{F}_{\mathrm{CO}, 0}^{\mathrm{R}}-\mathrm{F}_{\mathrm{CO}}^{\mathrm{R}}}{\mathrm{F}_{\mathrm{CO}, 0}^{\mathrm{R}}}
$$

La cual es válida debido a que el monóxido de carbono no permea hacia los tubos.

- El cociente entre la cantidad permeada de hidrógeno y la cantidad máxima de hidrógeno que puede ser obtenida (Índice de Permeación), que representa la fracción de hidrógeno global que permea a través de la membrana cuando todo el monóxido de carbono reacciona (ver ecuación 27 ).

$$
\mathrm{IP}_{\mathrm{H}_{2}}=\frac{\mathrm{F}_{\mathrm{H}_{2}}^{\mathrm{P}}}{\mathrm{F}_{\mathrm{H}_{2}}^{\mathrm{R}}+\mathrm{F}_{\mathrm{CO}}^{\mathrm{R}}}
$$

Para todos los casos de estudio se utilizó un volumen de catalizador de $2260 \mathrm{~cm}^{3}$ y una relación de longitud de reactor y diámetro interno de coraza igual a 2 , según lo propuesto por Adrover et al. Los tubos poseen un diámetro interno y externo de 13.4 y $8 \mathrm{~mm}$, respectivamente [6].El número de tubos se fija entre 1 y 60, y se calcula el diámetro interno de la coraza. Los resultados se muestran en la Fig. 3.1 y 3.2. 


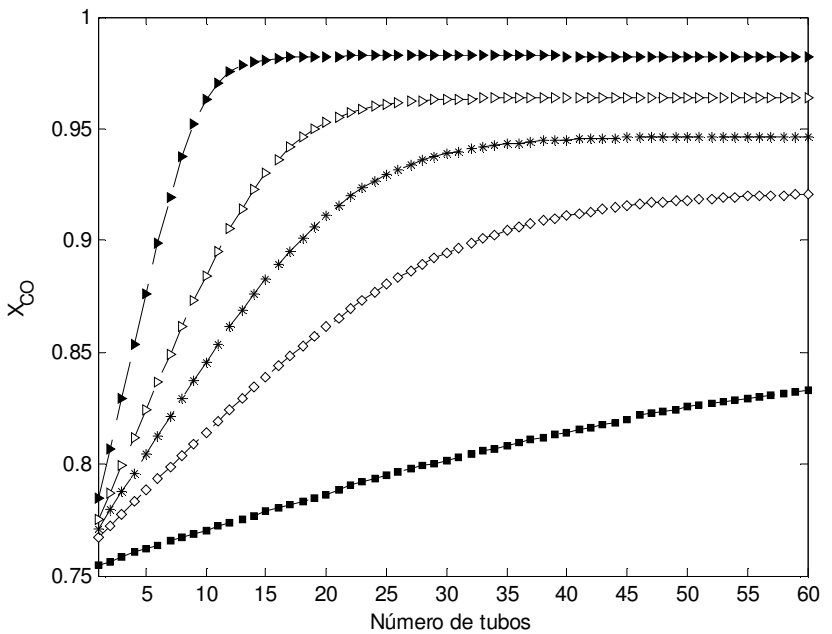

Figura 3.1. Conversión del monóxido de carbono contra número de tubos a diferentes valores de presión en el retentato

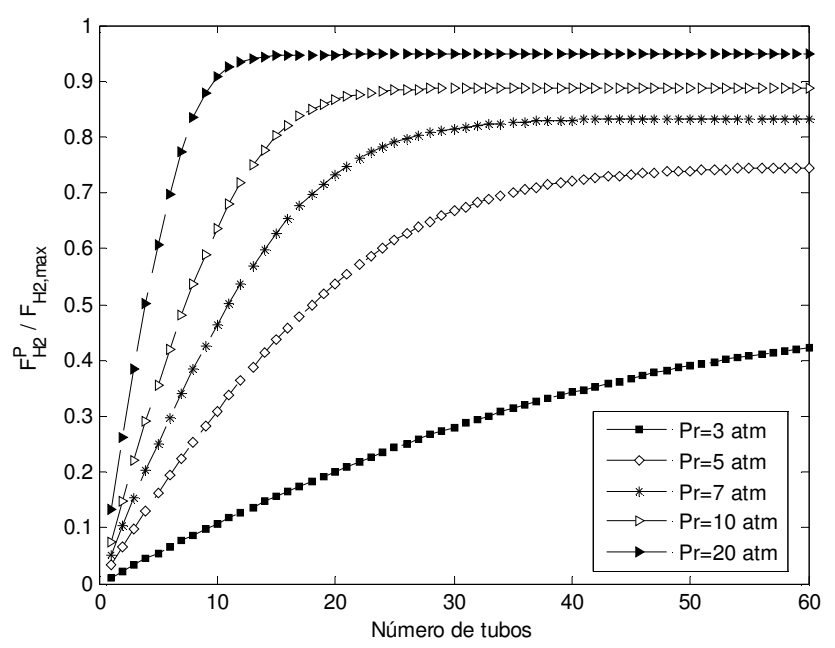

Figura 3.2. Índice de permeación contra número de tubos a diferentes valores de presión en el retentato

Los dos perfiles, tanto el de conversión como índice de permeación presentan claras similitudes en las tendencias para los valores de presión en el retentato y número de tubos. Además, el aumento del número de tubos permite una mayor área de permeación para el hidrógeno, por lo que el flujo de este hacia el permeato aumentará proporcionalmente. Para todos los valores de presión en el retentato (exceptuando el valor de $3 \mathrm{~atm}$ ), se alcanza y se estabiliza un valor máximo en la conversión y el índice de permeación. Se analizan a continuación los valores de presión de retentato que producen mayores valores de conversión e índice de permeación, según la metodología de Moon y Park. Se observa que para los valores de presión de 10 y 20 atm, la cantidad de tubos en los que no hay cambios significativos son entre aproximadamente 20 y 30 tubos, respectivamente. En las Fig. 4.1 y 4.2, se muestran las guías de diseño comparadas con las condiciones propuestas por Adrover et al., para las condiciones descritas de presión utilizando para ambos casos un valor de 20 tubos.

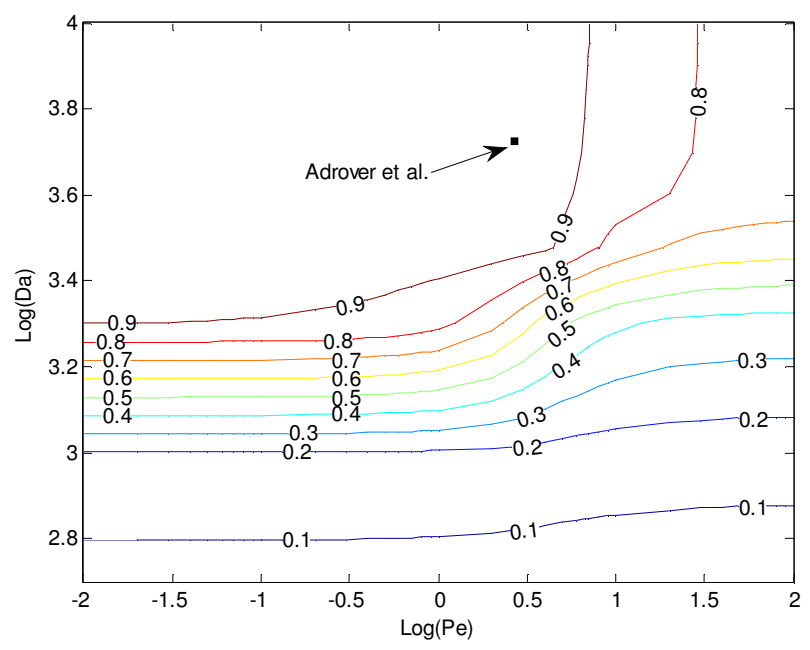

Figura 4.1. Conversión de salida como función de $P e$ y $D a$ para el caso 1: $P^{R}=10$ atm y $N_{t}=20$

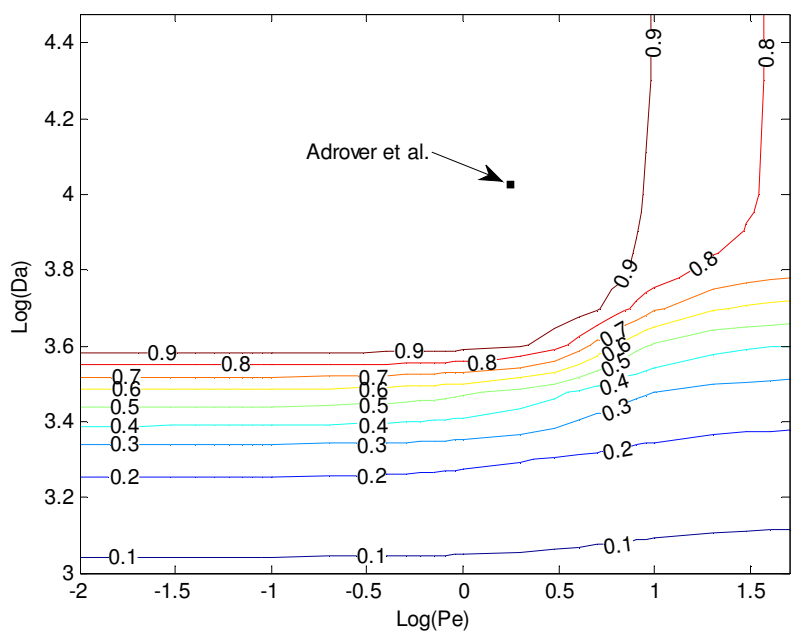

Figura 4.2. Conversión de salida como función de Pe y Da para el caso 2: $\mathrm{P}^{\mathrm{R}}=20$ atm y $\mathrm{N}_{\mathrm{t}}=20$

Los valores de conversión de salida del RM más elevados se encuentran para valores altos de Damköehler, y bajos para el caso del número de Peclet. La permeación de hidrógeno a través de la membrana aumenta con valores bajos de Pe, lo que resulta, en un desplazamiento del equilibrio en favor de los productos. Las condiciones de operación propuestas por Adrover et al., se ubican en la región mencionada, donde se alcanzan conversiones del $95.16 \%$ y $97.86 \%$ para cada uno de los casos planteados respectivamente. La diferencia entre la cantidad de hidrógeno obtenida por el lado del permeato para los casos analizados, alcanza un $6.87 \%$ (a favor del caso 2). De esta forma, un numero de tubos de 20 junto con una presión de retentato de 20 atmósferas es una opción 
interesante, considerando que este tipo de membranas soporta una presión de alrededor de 16 atm según Brunetti et al [9].

Con las condiciones de operación definidas, se propone un arreglo cuadrado para los tubos del reactor, cuyas características se presentan en la Tabla 3.

\begin{tabular}{|c|c|}
\hline Parámetro de Diseño & Valor \\
\hline Diámetro Interno Coraza & $12.35 \mathrm{~cm}$ \\
\hline $\begin{array}{c}\text { Espaciado entre tubos } \\
{[10]}\end{array}$ & $2.54 \mathrm{~cm}$ \\
\hline Longitud Reactor & $24.69 \mathrm{~cm}$ \\
\hline
\end{tabular}

Tabla 3. Dimensiones del reactor de membrana multitubular, con

$$
P^{R}=20 \mathrm{~atm}, N_{t}=20
$$

Los perfiles de temperatura tanto para la coraza como tubos para estas condiciones, se presentan en la Fig. 5.

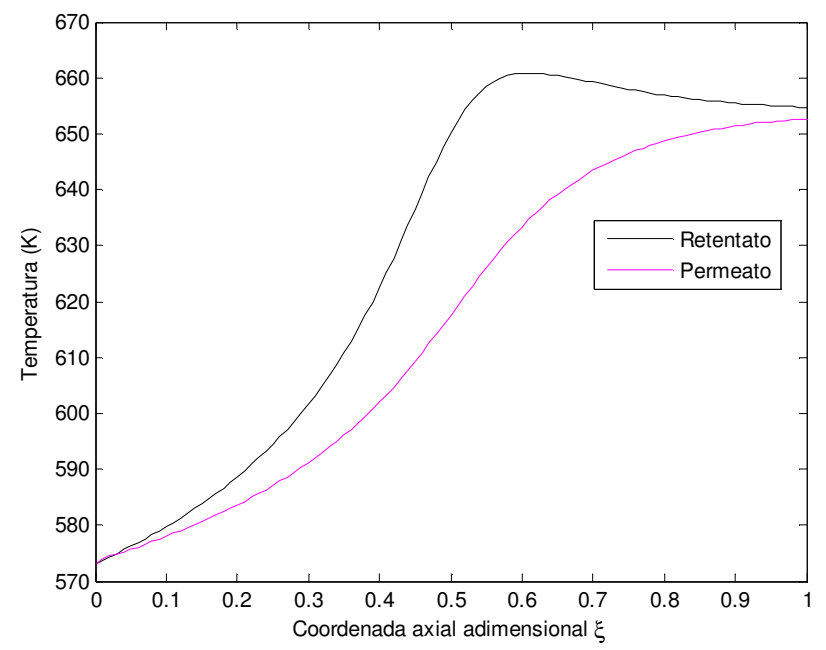

Figura 5. Perfiles de temperatura para el lado del permeato y retentato, con $\mathrm{P}^{\mathrm{R}}=20 \mathrm{~atm}$ y $\mathrm{N}_{\mathrm{t}}=20$

La diferencia de temperatura entre coraza y tubos es moderadamente baja, principalmente por que la transferencia por convección es buena, soportada por valores de U relativamente altos (entre $45-58 \mathrm{~W} / \mathrm{m}^{2} \mathrm{~K}$ ). A la salida del reactor, las corrientes salen aproximadamente a una temperatura de $653 \mathrm{~K}$, donde no se observan cambios abruptos entre estas.

A continuación se compara el sistema con un reactor convencional usando la misma cantidad de catalizador $\left(2260 \mathrm{~cm}^{3}\right)$, donde los balances se diferencian puesto que el flujo de permeación de hidrógeno es igual a cero. Se realiza el cálculo para iguales presiones de trabajo del RM como se muestra en la Fig 6.

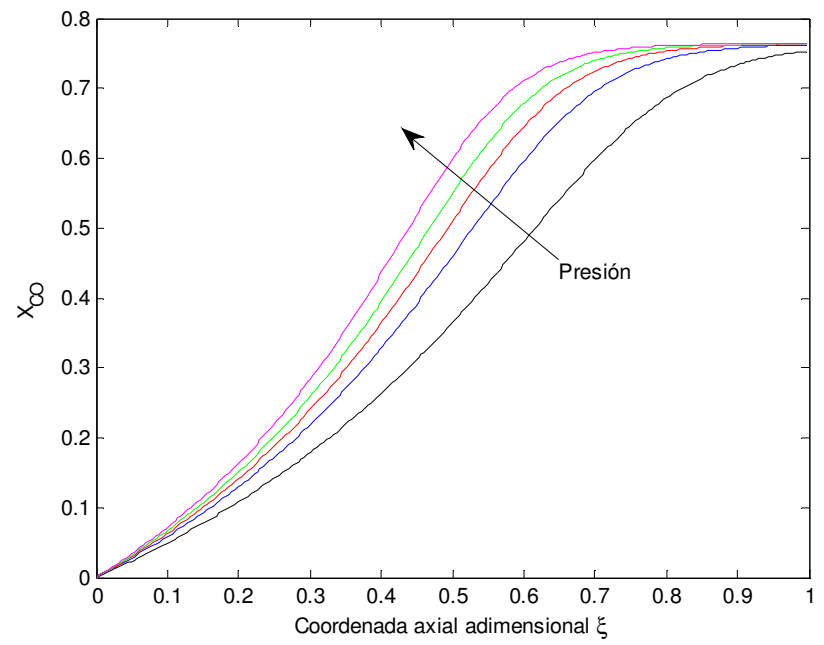

Figura 6. Conversión del monóxido de carbono contra longitud adimensional para presiones de 3, 5, 7, 10 y 20 atm

Aunque los caminos realizados para cada configuración de reactor son distintos, al final se obtiene aproximadamente la misma conversión de salida, lo que muestra que no hay efecto significativo de la presión de trabajo. El valor obtenido de conversión alcanza un $76 \%$ para todos los casos, es decir, un $22 \%$ menor comparada con la configuración propuesta para el RM, lo que demuestra la importancia de retirar progresivamente productos para este tipo específico de reacción. Además, en el RM se obtiene una corriente pura de hidrógeno que permite su uso posterior directamente en las celdas de combustible, y no tiene que ser sometida a un proceso de separación posterior.

\section{CONCLUSIONES}

Se encuentra que el reactor de membrana multitubular presenta altas conversiones para la producción de hidrógeno al ser comparado con un reactor convencional (mayor en un $22 \%$ ). Esto se debe principalmente a que el RM proporciona las condiciones para el desplazamiento del equilibrio, por la separación gradual del hidrógeno alimentado y producido.

La conversión del monóxido de carbono y la permeación son sensibles tanto para cambios en la presión en el retentato como en el número de tubos. Para ambos casos, la conversión y permeación aumentan con el incremento del parámetro de diseño, puesto que se provee un alto gradiente de concentración entre tubos y coraza, así como un aumento del área de transferencia de masa.

Aunque en este trabajo se propone un modelo tentativo de un reactor multitubular, deben considerarse análisis de costos, el sistema global y condiciones de los materiales del equipo (e.g. presión máxima de operación de la membrana), para obtener un diseño final apropiado y realista. 


\section{REFERENCIAS}

[1]. P. Lutze, R. Gani, J.M. Woodley, "Process intensification: A perspective on process synthesis," Chem. Eng. and Processing: Process Intensification. [Online]. vol. 49, pp. 547-558, 2010. Available: http://www.sciencedirect.com/science/article/pii/S02 55270110001182

[2]. J.A. Francesconi, M.C. Mussati, P. Aguirre, "Analysis of design variables for water gas-shift reactors by model-based optimization," Journal of Power Sources. [Online]. vol. 173, pp. 467-477, 2007. Available:

http://www.sciencedirect.com/science/article/pii/S03 78775307008063

[3]. J. Coronas, J. Santamaría, "Catalytic reactors based on porous ceramic membranes," Catalysis Today. [Online]. vol. 51, pp. 377-389, 1999. Available: http://www.sciencedirect.com/science/article/pii/S09 20586199000905

[4]. S.A.S Rezai, Y. Traa, "Equilibrium shift in membrane reactors: A thermodynamic analysis of the dehydrogenative conversion of alkanes," J. Memb. Sci. [Online]. vol. 319, pp. 279-285, 2008. Available: http://www.sciencedirect.com/science/article/pii/S03 7673880800272X

[5]. W.S. Moon, S.B. Park, "Design guide of a membrane for a membrane reactor in terms of permeability and selectivity," J. Memb. Sci. [Online]. vol. 170 pp. 4351, 2000. Available:

http://www.sciencedirect.com/science/article/pii/S03 76738899003622

[6]. M.E. Adrover, E. López, D.O. Borio, M.N. Pedernera, "Simulation of a membrane reactor for the WGS reaction: Pressure and thermal effects," A.I.Ch.E. Journal. [Online]. vol. 154, pp. 196-202, 2009. Available:

http://www.sciencedirect.com/science/article/pii/S13 85894709003271

[7]. W.F. Podolski, Y.G. Kim, "Modeling the water-gas shift reaction," Industrial and Engineering Chemistry. [Online]. vol. 13 (4), pp. 415-421, 1974. Available:

http://pubs.acs.org/doi/abs/10.1021/i260052a021

[8]. F. Scura, G. Barbieri, E. Drioli, "H2 for PEM-FC: effect of $\mathrm{CO}$ in the purification by means of Pd-based membranes," Desalination. [Online]. vol. 200, pp. 239-241, 2006. Available:

http://www.sciencedirect.com/science/article/pii/S00 $1191640600957 \mathrm{X}$

[9]. A. Brunetti, A. Caravella, G. Barbieri, E. Drioli, "Simulation study of water gas shift reaction in a membrane reactor," Journal of Membrane Science. [Online]. vol. 306 pp. 329-340, 2007. Available: http://www.sciencedirect.com/science/article/pii/S03 $\underline{76738807006564}$
[10]. D.Q. Kern, Procesos de Transferencia de Calor, vol. XXXI. Editorial CECSA 1999.

[11]. G.F. Froment, K.B. Bischoff, J. de Wilde, Chemical Reactor Analysis and Design, vol III. John Wiley \& Sons, Inc 2011. 